of the reproduced sound is good, but it deteriorates rapidly. The system used by the Post Office is the photographic system, and photocells are used. The technique is complicated, but the record is not subjected to any mechanical wear and is therefore, for all practical purposes, permanent.
During the first week of the public operation of the service, nearly 400,000 calls were made, and during each subsequent week the number has remained practically constant at about 200,000 . The relay sets at present permanently installed can each supply 100 simultaneous calls.

\title{
Noise on the Road
}

$\mathrm{T}$ HE Departmental Committee on "Noise in the Operation of Mechanically Propelled Vehicles" has published its third interim report (H.M. Stationery Office, $1 s$. net). The Committee, of which Dr. G. W. C. Kaye is chairman, was set up by the Minister of Transport in 1934. The present report is based on loudness tests carried out for the Committee by the National Physical Laboratory on some 600 'used' motor-vehicles representative of all the main types on the roads to-day, and of various ages up to thirteen years. A comparison of the results with those from the earlier tests on new vehicles, shows that on the average the used vehicles were the louder by some $4-5$ phons. In the range of noise met with on the road (about 70-105 phons) ordinary motorcars were much the quietest vehicles, while amongst the loudest were certain motor-cycles and sports cars, particularly at high speeds.

The noise from the engines of ordinary cars and commercial vehicles is louder than the exhaust noise, but with motor-cycles and sports cars the position is reversed. This fact lends support to the Committee's earlier criticism of the unsatisfactory nature of the silencing of many motor-cycles and sports cars, and the investigation generally indicates that greater progress has been made in the silencing of sports cars than of motor-cycles.

In its previous reports the Committee made the recommendation that new motor-vehicles should not be offered for sale if the loudness under prescribed conditions of test exceeded 90 phons, adding that motor-cycles should be allowed a latitude of 5 phons for two years. The Committee now proposes for vehicles actually in use on the road a limit of 95 phons (that is, about the noise in a tube train). No vehicles would be allowed on the highway if the loudness, when measured at a point $18 \mathrm{ft}$. laterally from the middle of the vehicle or $25 \mathrm{ft}$. behind the open end of the exhaust pipe, exceeded this limit in a normal running test and in a racing engine test conducted in the same way as for newly manufactured vehicles. The Committee also recommends that this limitation of loudness should be applied at first only to vehicles first registered after an appointed day, but that two years thereafter it should apply to vehicles of any age.

It will be appreciated that the noise limit proposed, while making very modest demands on the majority of manufacturers and owners of motor-vehicles, would, by ruling out the arch offenders, form a substantial contribution to the amenities of the road. It is to be hoped, moreover, in the public interest that future developments will enable the limit to be lowered as time goes on. At the Minister's request, the Committee is now investigating the noise caused by motor horns with the view of advising him whether action might usefully be taken to abolish the more strident noises of this kind. The question of the noise arising from trams and trolley buses is under examination by the Ministry in conjunction with the associations concerned.

\section{Hirosima Wave Geometry and Field Theory}

$\mathrm{R}$ EFERENCE has already been made in this journal (Dec. 21, 1935) to the vigorous school of mathematical physics now flourishing in the Hirosima University, Japan. These investigations are continuing (J. Sci. Hirosima Univ., A, 7 ; 1937), bringing the number of memoirs on the subject up to fiftcen. A good summary of the whole series is contained in the paper "Geometrization of the Law of Physics" (ibid., p. 81) by Y. Mimura.

It is claimed that the new theory bears a relation to Einstein's theory similar to that which Einstein's bears to Newton's, or to that which modern quantum mechanics bears to the old quantum theory. Einstein's theory is described as semi-geometrical, and the same term is applied to all existing unified field theories, as they are all built by a mixture of two procedures, namely, the finding of an appropriate geometry, and the finding of an appropriate invariant density to be used with a generalized Hamilton's principle, which is assumed to be the funda. mental law of physics.

The Hirosima school considers that the generalized
Hamilton's principle should be dispensed with, and they replace it by the condition of the complete integrability of the fundamental differential equation, which is obtained by purely geometrical means. In the hope that 'wave geometry' is identical with physics, extensive investigations of possible varieties have been carried out, including those corresponding to four-dimensional ordinary metrical geometry, to projective geometry, and to conformal geometry.

It is acknowledged that the question as to which wave geometry is to be identified with physies can only be judged by its results. The new field theory has already succeeded in deducing some important physical results, such as a gravitional wave in Maxwellian form, a line element of de Sitter's form, and one equivalent to Schwarzschild's. Perhaps it is not unfair to say that, in these days of competing unified field theories, the ordinary physicist must be allowed to suspend judgment until the physical consequences of the theories have been worked out very much more fully than has been done at present. 